\title{
Number-parity effect for confined fermions in one dimension
}

\author{
Christian Schilling ${ }^{1, *}$ and Rolf Schilling $2, \dagger$ \\ ${ }^{1}$ Clarendon Laboratory, University of Oxford, Parks Road, Oxford OX1 3PU, United Kingdom \\ ${ }^{2}$ Institut für Physik, Johannes Gutenberg-Universität Mainz, Staudinger Weg 9, D-55099 Mainz, Germany \\ (Received 19 August 2015; revised manuscript received 25 September 2015; published 1 February 2016)
}

\begin{abstract}
For $N$ spin-polarized fermions with harmonic pair interactions in a one-dimensional trap an odd-even effect is found. The spectrum of the one-particle reduced density matrix of the system's ground state differs qualitatively for $N$ odd and $N$ even. This effect only occurs for strong attractive and repulsive interactions. Since it does not exist for bosons, it must originate from the repulsive nature implied by the fermionic exchange statistics. In contrast to the spectrum, the one-particle density and correlation function for strong attractive interactions do not show any sensitivity on the number parity. This also suggests that reduced-density-matrix-functional theory has a more subtle $N$ dependency than density-functional theory.
\end{abstract}

DOI: 10.1103/PhysRevA.93.021601

Introduction. Physical behavior often depends qualitatively on binary parameters as, e.g., odd and even or integer and half-integer. Such parity effects play an important role in physics. A well-known example is Kramers' number-parity effect, i.e., the twofold degeneracy of the eigenstates of a quantum system with an odd number of electrons, provided time-reversal symmetry holds [1]. Haldane [2] has shown the existence of a spin-parity effect. The spectrum of the quantum Heisenberg antiferromagnet in one dimension has an energy gap for all integer spins, whereas it is gapless in the case of half-integer spins. Recently, an interesting number-parity effect has been observed experimentally for a few ultracold fermions in a quasi-one-dimensional trap. Tuning the potential such that the pair interactions become attractive, Cooper pairs are formed. Their tunneling is different for an odd and an even number of fermions [3]. Based on Kramers' theorem another number-parity effect was proven to exist for fermionic one-particle reduced density matrices (1-RDM) [4] (see also Ref. [5]). There it was shown that the eigenvalues of a 1-RDM arising from an eigenstate of a time-reversal symmetric Hamiltonian are twofold degenerate for an even number of fermions.

In the present work we will show that the so-called natural occupation numbers, i.e., the spectrum $\left\{\lambda_{k}\right\}$ of the ground state 1-RDM for strongly attractive and spin-polarized fermions confined in one dimension also exhibits an odd-even effect. Since the spin-polarizing magnetic field breaks time-reversal symmetry, this effect is completely different from that found in Ref. [4]. Furthermore, it does not occur for bosons. Consequently, it must result from the fermionic exchange symmetry.

Besides the relevance of parity effects on their own, exploring the structure of reduced density matrices has also gained a lot of relevance during recent years. This is essentially due to progress [6-11] in the quantum marginal problem (QMP) which studies the relation of reduced density matrices arising from a common multipartite quantum state. For basic overviews of the QMP the reader may consult Refs. [12-14]. The most prominent QMP is the two-particle

\footnotetext{
*christian.schilling@physics.ox.ac.uk

†rschill@uni-mainz.de
}

$N$-representability problem [5,15], the description of 2-RDM arising from $N$-fermion quantum states. Its solution would allow one to efficiently calculate ground states of fermionic quantum systems with two-body interactions. However, since this problem and most of the other QMPs are quantum Merlin-Arthur hard [16], already partial insights on the set of compatible density matrices are highly appreciated and alternative methods for the ground state calculation are gaining importance as well. One such promising method is reduced-density-matrix-functional theory (see, e.g., $[17,18]$ ). This natural extension of density-functional theory [19] seeks a distinguished functional $\mathcal{F}$ on the 1-RDM whose minimization leads to the exact ground state energy and the corresponding ground state 1-RDM. Any structural insights on ground state 1-RDM contributes to this task of finding or approximating $\mathcal{F}$ by exposing further necessary constraints on legitimate functionals.

Model and 1-RDM. We consider $N$ identical particles with mass $m$ in a one-dimensional harmonic trap interacting via a harmonic two-body potential. If $x_{i}$ is the position of the $i$ th particle the Hamiltonian reads

$$
\hat{H}=\sum_{i=1}^{N}\left[-\frac{\hbar^{2}}{2 m} \frac{\partial^{2}}{\partial x_{i}^{2}}+\frac{1}{2} m \omega^{2} x_{i}^{2}\right]+\frac{1}{2} D \sum_{1 \leqslant i<j \leqslant N}\left(x_{i}-x_{j}\right)^{2},
$$

where $\omega$ is the eigenfrequency of the trap and $D$ is the interaction strength, which can be positive or negative. Stability requires $D>D_{\text {low }} \equiv-m \omega^{2} / N$.

Hamiltonian (1) arises as an effective model, e.g., for the description of quantum dots, where the Coulomb interaction between the electrons is screened (see, e.g., Ref. [20]). Furthermore, it was used to understand the emergence of shell structures in atoms (see, e.g., Ref. [21]) and nuclei (see, e.g., Ref. [22]).

The great advantage of model (1) is the exact knowledge of all its eigenstates [23-27]. For arbitrary numbers of bosons and any spatial dimension the ground state 1-RDM can easily be calculated [24-27]. Based on such analytic results BoseEinstein condensation in harmonic traps was explored (see, e.g., Ref. [28]). In contrast to bosons, the analytical calculation of the corresponding fermionic 1-RDM is much more involved. 
We will show that the properties of the fermionic 1-RDM are much richer, compared to the bosonic case, leading to new insights.

For spin-polarized (or spinless) fermions in one dimension, the spatial part of the ground state of Hamiltonian (1) is the totally antisymmetric wave function [23-25] (see also Refs. [20,21])

$$
\begin{aligned}
\Psi_{0}^{(f)}\left(x_{1}, \ldots, x_{N}\right)= & \mathcal{N}_{N}^{(f)}\left[\prod_{1 \leqslant i<j \leqslant N}\left(x_{i}-x_{j}\right)\right] \\
& \times \exp \left[-A\left(x_{1}^{2}+\cdots+x_{N}^{2}\right)\right. \\
& \left.+B\left(x_{1}+\cdots+x_{N}\right)^{2}\right]
\end{aligned}
$$

with $\mathcal{N}_{N}^{(f)}$ a normalization factor and

$$
A=\frac{1}{2 l_{+}^{2}}, \quad B=\frac{1}{2 N}\left(\frac{1}{l_{+}^{2}}-\frac{1}{l_{-}^{2}}\right) .
$$

$l_{-}=\sqrt{\hbar / m \omega}$ and $l_{+}=\sqrt{\hbar / m\left(\omega^{2}+D N / m\right)^{1 / 2}}$ are the length scales for the center of mass and the relative motion, respectively. Note that $\Psi_{0}^{(f)}\left(x_{1}, \ldots, x_{N}\right)$ resembles Laughlin's wave function [29] for the fractional quantum Hall effect. We will come back to this point below.

The 1-RDM of wave function (2) follows as [24,25]

$$
\rho_{1}^{(f)}(x ; y)=\mathcal{N}^{(f)} F(x, y) \exp \left[-a\left(x^{2}+y^{2}\right)+b x y\right]
$$

with the polynomial $F(x, y)$ in $x$ and $y$ of degree $2(N-1)$ [30] and

$$
a=A-B-b / 2, \quad b=(N-1) B^{2} /[A-(N-1) B] .
$$

The factor $\mathcal{N}^{(f)}$ (not to be confused with $\mathcal{N}_{N}^{(f)}$, the normalization constant of $\Psi_{0}^{(f)}$ ), follows from the normalization $\int_{-\infty}^{\infty} d x \rho_{1}^{(f)}(x ; x)=N$. The bosonic and fermionic 1-RDM are related by [25]

$$
\rho_{1}^{(f)}(x ; y)=\tilde{\mathcal{N}}^{(f)} F(x, y) \rho_{1}^{(b)}(x ; y),
$$

where $\tilde{\mathcal{N}}^{(f)}=\mathcal{N}^{(f)} / \mathcal{N}^{(b)}$ and $\mathcal{N}^{(b)}$ the corresponding normalization constant appearing in $\rho_{1}^{(b)}(x ; y)$. Consequently, the fermionic nature of $\rho_{1}^{(f)}(x ; y)$ is only contained in the polynomial prefactor $F(x, y)$. It arises from the polynomial prefactor of the exponential function in Eq. (2), the Vandermonde determinant, which is a result of the fermionic exchange symmetry.

The spectrum $\left\{\lambda_{k}\right\}$ follows from solving the eigenvalue equation

$$
\int_{-\infty}^{\infty} d y \rho_{1}^{(f)}(x ; y) \chi_{k}^{(f)}(y)=\lambda_{k}^{(f)} \chi_{k}^{(f)}(x),
$$

$k=1,2,3, \ldots$. In quantum chemistry the $\lambda_{k}$ are called natural occupation numbers. These eigenvalues will be ordered decreasingly, i.e., $\lambda_{k} \geqslant \lambda_{k+1}$ for all $k \geqslant 1$. Due to the duality $\left\{\lambda_{k}\left(l_{-}, l_{+}\right)\right\}=\left\{\lambda_{k}\left(l_{+}, l_{-}\right)\right\}$, first observed in Ref. [31] and proven in Ref. [32], we restrict to $l_{+} / l_{-} \leqslant 1$. Note, $l_{+} / l_{-}<1$ $\left(l_{+} / l_{-}>1\right)$ means attractive (repulsive) pair interactions. Since we are interested only in fermions we also suppress the superscript $(f)$.
Strong-coupling limit and results. For attractive interaction the strong-coupling limit $D N / m \omega^{2} \rightarrow \infty$ corresponds to the limit $t \equiv l_{+} / l_{-} \rightarrow 0$, performed at $N$ fixed. For repulsive interactions it follows for $D N / m \omega^{2} \rightarrow D_{\text {low }} N / m \omega^{2}$, corresponding to $t \rightarrow \infty$.

We will prove that completely unexpected features of the spectrum of the fermionic 1-RDM occur in this limit, which can be discussed analytically. Due to the duality property [32] we can restrict to attractive interactions, i.e., to $t \leqslant 1$. Technical details can be found in [30].

In the following all coordinates $x_{i}$ and all lengths will be measured in units of $l_{-}$. For $t \rightarrow 0$, there are three basic observations. First, the leading order of $\rho_{1}(x ; y)$ is proportional to $\exp \left\{-\frac{N-1}{4 N}[(x-y) / t]^{2}\right\}[30]$. Therefore, the weight of the deviation of $y$ from $x$ decreases extremely fast for decreasing $t$. Second, $\chi_{k}(x)$ varies on an $x$ scale proportional to $\sqrt{t}$ and third, the polynomial prefactor $F(x, y)$ converges to a polynomial $\tilde{F}(\tilde{z})$ where $\tilde{z}=(x-y) / t$ [30]. Let us introduce the rescaled variable $\tilde{x}=x / \sqrt{t}$ and $\chi_{k}(\sqrt{t} \tilde{x})=\exp \left(\frac{1}{2} N t \tilde{x}^{2}\right) \zeta_{k}(\tilde{x})$. Then, by using the momentum representation the eigenvalue equation (7) for $t \ll 1$ reduces to a Schrödinger equation (with position and momentum exchanged) [30]

$$
\left[-\frac{\hbar^{2}}{2 \tilde{m}} \frac{\partial^{2}}{\partial \tilde{p}^{2}}+V_{N}(\tilde{p})\right] \tilde{\zeta}_{k}(\tilde{p})=\left(-\lambda_{k}\right) \tilde{\zeta_{k}}(\tilde{p}) .
$$

Here $\tilde{\zeta}_{k}(\tilde{p})$ is the Fourier transform of $\zeta_{k}(\tilde{x})$,

$$
\tilde{m}_{N}=\hbar^{2} /\left[-2 t N V_{N}(0)\right]
$$

is the mass of the "particle," and

$$
V_{N}(\tilde{p})=-t \mathcal{N} \int_{-\infty}^{\infty} d \tilde{z} \tilde{F}(\tilde{z}) \cos (\sqrt{t} \tilde{p} \tilde{z}) \exp \left(-\frac{N-1}{4 N} \tilde{z}^{2}\right)
$$

is an effective potential, where the scaled momentum $\tilde{p}=$ $\sqrt{t} p / \hbar$ has been introduced. $p$ is the conjugate momentum of $x . V_{N}(\tilde{p})$ is a product of a polynomial in $(\sqrt{t} \tilde{p})^{2}$ of degree $(N-1)$ (originating from the fermionic nature of the 1-RDM) and a Gaussian $\exp \left[-\frac{N}{N-1}(\sqrt{t} \tilde{p})^{2}\right][30]$.

Figure 1 depicts $V_{N}(\tilde{p})$ for $N=3$ and $N=4$. One observes that its number of minima equals $N$. Since $V_{N}(\tilde{p})$ for arbitrary $N$ is symmetric, one of the minima must be at $\tilde{p}=0$, for $N$ odd. In this case it is the global minimum, which we checked systematically up to $N=19$ and for some exemplary $N$ up to $N=101$. For $N$ even, the global minimum is twofold degenerate, which we checked for $N=2,4,6, \ldots, 20$ and for some exemplary $N$ up to $N=100$. There is little doubt that these properties hold for all $N$. This qualitatively
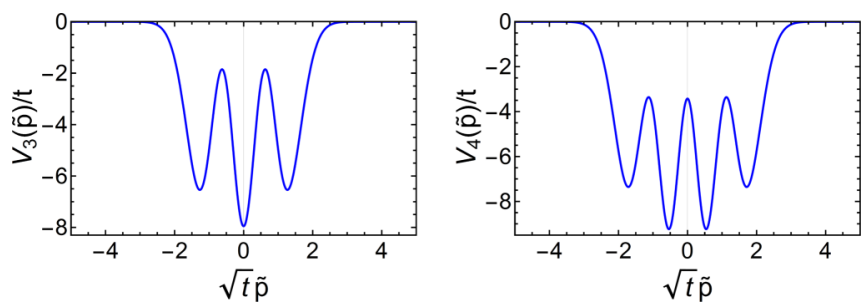

FIG. 1. Scaled effective potential as a function of $\sqrt{t} \tilde{p}$ for $N=3$ (left panel) and $N=4$ (right panel). 
different behavior of $V_{N}(\tilde{p})$ implies that the spectrum $\left\{\lambda_{k}\right\}$ of the ground state 1-RDM will qualitatively differ for an odd and even number of particles. Without solving the eigenvalue equation (8) one can already predict that the spectrum $\sigma$ of the $1-\mathrm{RDM}$ consists of two parts $\sigma^{\text {(up) }}$ for $k<k_{*}(t)$ and $\sigma^{\text {(low) }}$ for $k>k_{*}(t)$ where $k_{*}(t) \sim 1 / t$ is the $k$ value for which $\left(-\lambda_{k}\right)$ equals the height of the highest maximum of $V_{N}(\tilde{p}) . \sigma^{\text {(low) }}$ consists of "isolated" eigenvalues only, and does not depend qualitatively on the number parity. In contrast, the upper part, $\sigma^{\text {(up) }}$, differs qualitatively for $N$ odd and even. For $N$ odd it consists of subsets with "isolated" eigenvalues and subsets of pairs of quasidegenerate eigenvalues, whereas for $N$ even only subsets with quasidegenerate eigenvalues occur.

This number parity effect can be illustrated by calculating analytically the largest eigenvalues of the 1-RDM corresponding to the low-lying eigenvalues $\left(-\lambda_{k}\right)$ of the "particle" in the effective potential. This will be done by use of the harmonic approximation $V_{N}(\tilde{p}) \simeq V_{N}^{(\min )}+\frac{1}{2} V_{N}^{\prime \prime(\min )}\left(\tilde{p}-\tilde{p}_{\text {min }}\right)^{2}$ for the global minimum. Then, Eq. (8) reduces to the eigenvalue equation of a harmonic oscillator with mass $\tilde{m}$, frequency $\tilde{\Omega}=\sqrt{V_{N}^{\prime \prime(\min )} / \tilde{m}}$, and eigenvalues $\bar{\lambda}_{k}=-\left(\lambda_{k}+V_{N}^{(\min )}\right)=$ $\hbar \tilde{\Omega}\left(k-\frac{1}{2}\right)$. Accordingly, we obtain

$$
\lambda_{k} \simeq t \alpha\left[1-t \beta\left(k-\frac{1}{2}\right)\right]
$$

for $k=1,2,3, \ldots$. The coefficients follow from

$$
\alpha=-V_{N}^{(\min )} / t, \quad \beta=\sqrt{2 N V_{N}^{\prime \prime(\min )} /\left(-t V_{N}^{(\min )}\right)},
$$

which are of $O\left(t^{0}\right)$.

For $N$ odd, the global minimum of $V_{N}(\tilde{p})$ is nondegenerate at $\tilde{p}=0$. Hence the smallest eigenvalues $\left(-\lambda_{k}\right)$ and therefore the largest eigenvalues $\lambda_{k}$ of Eq. (7) are "isolated." In the case of $N$ even, the global minimum is twofold degenerate such that the tunneling between both minima leads to a splitting $\left(\Delta \lambda_{k}^{+}+\right.$ $\left.\Delta \lambda_{k}^{-}\right)$. Therefore, the eigenvalues appear in quasidegenerate pairs $\left\{\lambda_{2 k-1}, \lambda_{2 k}\right\}$ given by

$$
\lambda_{2 k-1} \simeq t \alpha\left[1-t \beta\left(k-\frac{1}{2}\right)\right]+\Delta \lambda_{k}^{+}
$$

for $k=1,2,3, \ldots . \lambda_{2 k}$ follows from Eq. (13) by replacing $\Delta \lambda_{k}^{+}$by $\left(-\Delta \lambda_{k}^{-}\right)$.

The results (11) and (13) show that $\lambda_{k}$ is of $O(t)$. Its $k$ dependency is proportional to $t^{2}$. Since these eigenvalues are the occupancies of the one-particle states $\chi_{k}(x)$ they are non-negative. Hence, the validity of Eqs. (11) and (13) is limited to $k<k_{*} \sim 1 / t$. They are also based on the harmonic approximation, which restricts their validity even more (see below). Furthermore, the tunneling splitting $\left(\Delta \lambda_{k}^{+}+\Delta \lambda_{k}^{-}\right)$ can be estimated. Equation (10) shows that $V_{N}(\tilde{p})=O(t)$ and that it varies on a scale $1 / \sqrt{t}$. This implies $\tilde{m}=O\left(t^{-2}\right)$, a potential barrier $\Delta V_{N}=O(t)$, and a tunneling distance $\Delta \tilde{p}=O(1 / \sqrt{t})$. Using that the splitting is proportional to $\exp \left(-\sqrt{\tilde{m} \Delta V_{N}} \Delta \tilde{p} / \hbar\right)$ it follows that $\left(\Delta \lambda_{k}^{+}+\Delta \lambda_{k}^{-}\right) \sim$ $\exp [-O(1 / t)]$, i.e., for $N$ even the pairs $\left(\lambda_{2 k-1}, \lambda_{2 k}\right)$ become quasidegenerate in the regime of strong coupling.

In order to test these predictions we have determined the eigenvalues by solving numerically the original eigenvalue
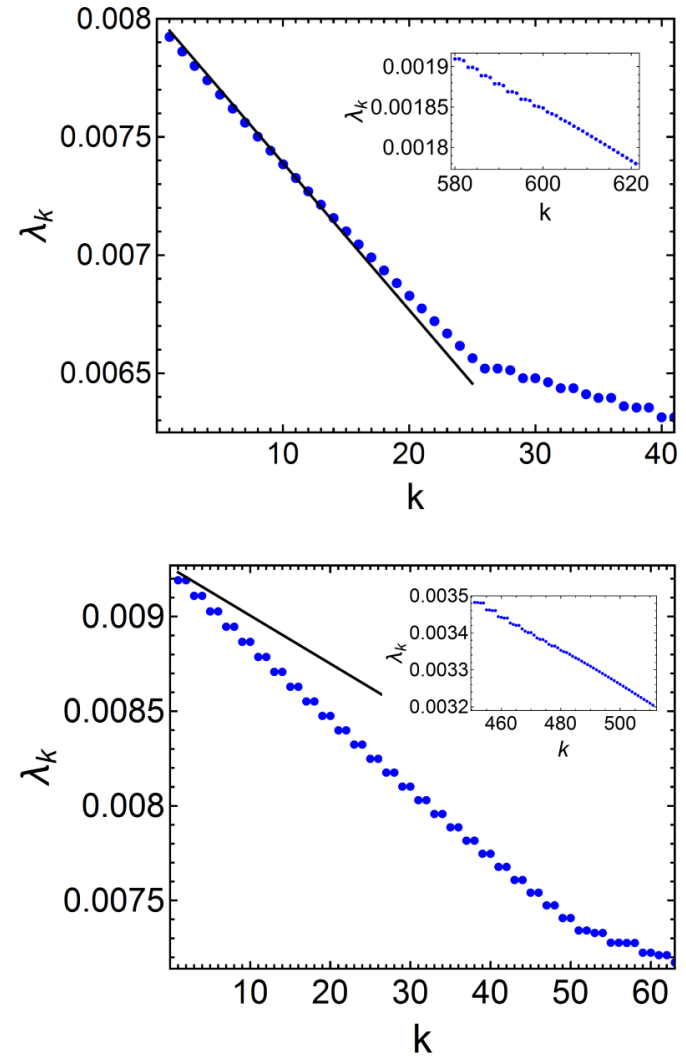

FIG. 2. Spectrum $\left\{\lambda_{k}\right\}$ for $t=10^{-3}$. Upper panel: $N=3$ and $1 \leqslant k \leqslant 40$; lower panel: $\quad N=4$ and $1 \leqslant k \leqslant 62$. The solid lines present the corresponding analytical result for $\left\{\lambda_{k}\right\}$ from Eq. (11) and from the first term of Eq. (13), respectively. The insets show the final crossover from the regime of quasidegenerate to that of nonquasidegenerate eigenvalues.

equation (7) for $N=3$ up to $N=8$. Note that $N=2$ is a special case, since there, all eigenvalues are automatically twofold degenerate as a Hamiltonian-independent consequence of the fermionic exchange statistics (see Theorem 4.1 in Ref. [5]).

Figure 2 presents the larger eigenvalues for the coupling $t=10^{-3}$ for $N=3$ and $N=4$. In the case of $N=3$ the subsequent eigenvalues have almost the same distance which is of $O\left(t^{2}\right)$, whereas for $N=4$ they occur in quasidegenerate pairs with $\lambda_{2 k-1}-\lambda_{2 k+1}=O\left(t^{2}\right)$ and $\lambda_{2 k-1}-\lambda_{2 k} \sim$ $\exp [-O(1 / t)]$, as predicted by our analysis above. Comparing for $N=3$ the numerical result with the analytical one, Eq. (11), shows very good agreement for $\lambda_{1}$ and the slope $d \lambda_{k} / d k$, for small $k$. For $N=4, \lambda_{1}$ is also well reproduced. However, the analytical and numerical values for the slope $d \lambda_{k} / d k$ deviate stronger from each other. This is a consequence of the fact that in contrast to $N=3$ the harmonic approximation is rather poor due to $\left(\tilde{p}-\tilde{p}_{\text {min }}\right)^{3}$ anharmonicities of $V_{4}$ around $\tilde{p}_{\text {min }}$. Note, our major achievement is not results (11) and (13) for the largest eigenvalues, but

(i) the qualitatively different spectrum of the 1-RDM for $N$ odd and even in the strong-coupling limit and

(ii) the generation and annihilation of quasidegenerate pairs of eigenvalues for those indices $k$ for which $\left(-\lambda_{k}\right)$ becomes equal to the height of corresponding minima and maxima, respectively, of $V_{N}(\tilde{p})$. 

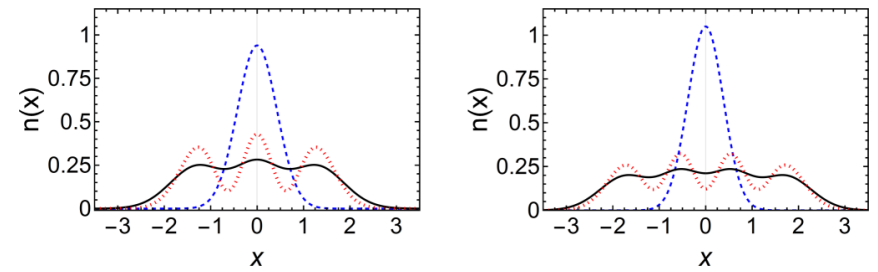

FIG. 3. $x$ dependency of the one-particle density for $N=3$ (left panel) and $N=4$ (right panel), where we set $l_{-} \equiv 1$. Blue dashed, black solid, and red dotted lines correspond to $t=0.1$ (attractive), $t=1$ (free fermions), and $t=10$ (repulsive), respectively [for $t=10$ it is plotted $10 n(10 x)]$.

Figure 2 demonstrates the creation of pairs of quasidegenerate eigenvalues and its inset illustrates their annihilation, e.g., at the highest maximum of $V_{N}(\tilde{p})$, where the crossover to "isolated" eigenvalues of $\sigma^{\text {(low) }}$ occurs.

Since under an increase of $N, V_{N}(\tilde{p})$ develops more and more extrema, there will be more and more regimes with groups of a different number of quasidegenerate pairs. In order to resolve these regimes for large $N, t$ must become small enough. Since the $t$ and $N$ dependence occurs as $t N$ [30] it must be $t \ll t_{*}(N) \sim 1 / N$. For macroscopically large $N$ of $O\left(10^{23}\right)$ this requires values for $t$ which are not realizable in experiments. Yet, for $N$ of $O\left(10^{2}\right)$, for which already macroscopic properties are present, this should be feasible. Accordingly, few-fermion systems are particularly suitable to observe these qualitative features of the spectrum of the 1-RDM.

$\lambda_{k}$ for $k \gg k_{*} \sim 1 / t$ can be determined analytically. With the approach discussed in Ref. [25] we obtain [30]

$$
\lambda_{k} \sim(k t)^{N-1} \exp \left[-\frac{2 N}{\sqrt{N-1}} t\left(k-\frac{1}{2}\right)\right],
$$

independent of the parity of $N$.

We have also calculated analytically the one-particle and two-particle densities $n(x) \equiv \rho_{1}(x ; x)$ and $n(x, y) \equiv$ $\rho_{2}(x, y ; x, y)$, respectively. The latter follows from the 2-RDM $\rho_{2}\left(x, y ; x^{\prime}, y^{\prime}\right) . n(x)$ is shown in Fig. 3 for $N=3$ and $N=4$. For noninteracting fermions (black solid line), the "layering" of the particles within the harmonic trap can be seen. This "shell structure" also exists for repulsive (red dotted line) and weak attractive coupling, but disappears completely for strong attractive interactions (blue dashed line), becoming qualitatively independent of $N$, which we checked up to $N=20$. The duality discussed in Ref. [32] implies that the one-particle density in momentum space for strong repulsive interaction behaves similarly as $n(x)$ for strong attractive interactions, i.e., it becomes structureless, as well. Quite similar behavior has been found for $n(x, y)$. As demonstrated by Fig. 4 the layering (not shown) for $n(x, y)$ disappears again for strong attractive coupling, and does not exhibit any qualitative sensitivity on $N$ (cf. left and right panels of Fig. 4). All these properties also hold for the correlation function $C(x, y)=n(x, y) / n(x) n(y)$.

Summary and conclusions. We have shown that the oneparticle description in the form of the 1-RDM exhibits an odd-even effect. The spectrum of the 1-RDM related to the fermionic ground state of our one-dimensional harmonic
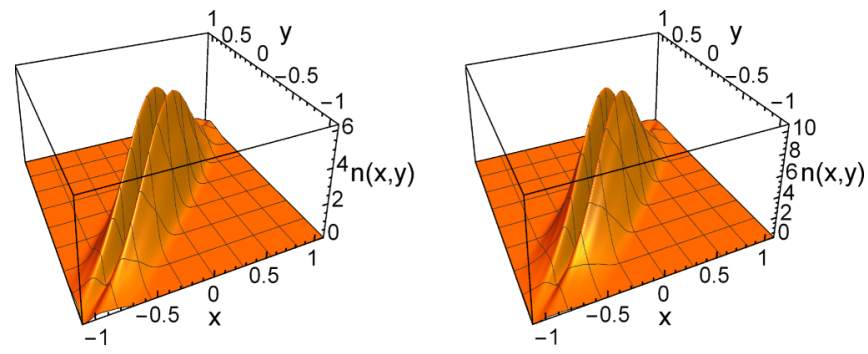

FIG. 4. $x$ and $y$ dependency of the two-particle density $n(x, y)$ (not normalized and we set $l_{-} \equiv 1$ ) for $t=0.1, N=3$ (left panel), and $N=4$ (right panel).

system differs qualitatively for an even and an odd number of particles. This effect only occurs for strong attractive and repulsive (due to the duality property [32]) interactions. The number-parity effect does not exist for bosons. Therefore it must originate from the repulsive nature implied by the fermionic exchange statistics (antisymmetry of the wave function). One may wonder how far the interplay between strong pair interactions (particularly for attractive ones) and the exchange symmetry leads to new phenomena for fermions, beyond the present parity effect. Also the investigation of the existence of the odd-even effect in more than one spatial dimension will be of interest.

It would also be interesting to develop tools which make it possible to investigate these predictions by experiments. In that case the parameter $t$ has to be tuned (see below) such that the splitting of the quasidegenerate eigenvalues is still large enough in order to be resolved. Since our model involves harmonic pair interactions one might be tempted to deny the relevance of our findings for realistic systems. There are two reasons why this might be not true. First, for arbitrary pair potential the particles in a trap form a one-dimensional lattice. Expanding the potential up to quadratic terms in the displacements with respect to the classical ground state will result in a harmonic model similar to that studied by us. This has recently been done for a one-dimensional $N$-particle system with long-range inverse power-law potential in order to calculate the von Neumann entanglement entropy [33]. Second, and even more important, the specific form of the pair interactions may not be as important as one might believe. As pointed out above, the odd-even effect for the spectrum of the fermionic 1-RDM is a result of the polynomial prefactor in Eq. (2) which makes the wave function totally antisymmetric. This requirement of antisymmetry has also been the guide leading to Laughlin's wave function for describing the fractional quantum Hall effect. This wave function again has a preexponential factor $\left[\prod_{1 \leqslant i<j \leqslant N}\left(z_{i}-\right.\right.$ $\left.z_{j}\right)^{p}$ ], with $p$ an odd natural number and $z_{i}$ the complex variable specifying the position of the $i$ th particle in the plane. Laughlin's ansatz is a surprisingly good approximation of the two-dimensional electron system's ground state, not only for a Coulombic pair potential but for harmonic interactions as well [29]. This suggests that the specific form of the pair interactions is less important, which is supported by the exact ground state solution of the one-dimensional CalogeroSutherland model [34-38]. Besides a harmonic trap potential this model contains pair interactions $g\left(x_{i}-x_{j}\right)^{-2}$. Its ground 
state involves a preexponential factor $\left[\prod_{1 \leqslant i<j \leqslant N}\left(x_{i}-x_{j}\right)^{p}\right]$ with $p(g)=\frac{1}{2}(1 \pm \sqrt{1+4 g})$. Choosing the coupling constant such that $p(g)$ is an odd natural number one obtains the ground state for $N$ fermions.

Therefore our results may also hold for ultracold fermionic atoms in an optical trap interacting by a contact potential [39-41]. The interaction can be tuned from attractive to repulsive. In particular, it can be made arbitrarily strong by approaching the Feshbach resonance. This would allow one to experimentally approach the strong-coupling limit discussed in the present Rapid Communication. Of course, another possibility to realize that limit is the decrease of the trap frequency. For instance, for ${ }^{7} \mathrm{Li}$ the trap frequency in the experimental setup in Ref. [42] is about 30 times smaller than in the setup of Ref. [43].

The odd-even effect may also initiate and guide a new direction in density and reduced-density-matrix-functional theory. Although three decades ago it had been argued that the $N$ dependency of the ground state energy implies an $N$ dependency of the density functionals $\mathcal{F}_{N}$ [44], all of the prominent functionals used today do not exhibit an explicit dependency on $N$ (see, e.g., [45]). The parity effect found by us is a demonstration of a subtle $N$ dependency of the spectrum of the ground state's 1-RDM, and therefore also of $\mathcal{F}_{N}$. In addition, the fact that the one-particle density $n(x)$ in the strong-coupling regime does not show any sensitivity on the number parity suggests that the $N$ dependence within reduced-density-matrix-functional theory $[17,18]$ is much more subtle than in ordinary density-functional theory [19].

Acknowledgments. We would like to thank D. Jaksch, S. Jochim, F. Schmidt-Kaler, P. van Dongen, and V. Vedral for helpful discussions. We are particularly grateful to $\mathrm{P}$. van Dongen for numerous valuable comments on our manuscript. C.S. gratefully acknowledges financial support from the Swiss National Science Foundation (Grant No. P2EZP2152190) and from the Oxford Martin Programme on Bio-Inspired Quantum Technologies.
[1] H. A. Kramers, Théorie générale de la rotation paramagnétique dans les cristaux, Proc. Amsterdam Acad. 33, 959 (1930).

[2] F. D. M. Haldane, Continuum dynamics of the 1-D Heisenberg antiferromagnet: Identification with the $\mathrm{O}(3)$ nonlinear sigma model, Phys. Lett. A 93, 464 (1983).

[3] G. Zürn, A. N. Wenz, S. Murmann, A. Bergschneider, T. Lompe, and S. Jochim, Pairing in Few-Fermion Systems with Attractive Interactions, Phys. Rev. Lett. 111, 175302 (2013).

[4] D. W. Smith, $n$-representability problem for fermion density matrices. II. The first-order density matrix with $n$ even, Phys. Rev. 147, 896 (1966).

[5] A. J. Coleman, Structure of fermion density matrices, Rev. Mod. Phys. 35, 668 (1963).

[6] A. Klyachko, Quantum marginal problem and representations of the symmetric group, arXiv:quant-ph/0409113v1.

[7] S. Daftuar and P. Hayden, Quantum state transformations and the Schubert calculus, Ann. Phys. (NY) 315, 80 (2005).

[8] A. Klyachko, Quantum marginal problem and n-representability, J. Phys.: Conf. Series 36, 72 (2006).

[9] M. Christandl and G. Mitchison, The spectra of quantum states and the Kronecker coefficients of the symmetric group, Commun. Math. Phys. 261, 789 (2006).

[10] M. Altunbulak and A. Klyachko, The Pauli principle revisited, Commun. Math. Phys. 282, 287 (2008).

[11] M. Altunbulak, The Pauli principle, representation theory, and geometry of flag varieties, Ph.D. Thesis, Bilkent University, 2008.

[12] C. Schilling, in The Qantum Marginal Problem, Mathematical Results in Quantum Mechanics (World Scientific, Singapore, 2014), Chap. 1, pp. 165.

[13] C. Schilling, Quantum marginal problem and its physical relevance, Ph.D. Thesis, ETH-Zürich, 2014, http://ecollection.library.ethz.ch/view/eth:8552.

[14] A. Lopes, Pure univariate quantum marginals and electronic transport properties of geometrically frustrated systems, Ph.D. Thesis, University of Freiburg, 2015, https://www.freidok.unifreiburg.de/data/10057.
[15] E. R. Davidson, Reduced Density Matrices in Quantum Chemistry (Academic, New York, 1976).

[16] Y.-K. Liu, M. Christandl, and F. Verstraete, Quantum Computational Complexity of the $n$-Representability Problem: QMA Complete, Phys. Rev. Lett. 98, 110503 (2007).

[17] T. L. Gilbert, Hohenberg-Kohn theorem for nonlocal external potentials, Phys. Rev. B 12, 2111 (1975).

[18] R. M. Erdahl and V. H. Smith, Density Matrices and Density Functionals (D. Reidel Publishing Company, Dordrecht, 1987).

[19] P. Hohenberg and W. Kohn, Inhomogeneous electron gas, Phys. Rev. 136, B864 (1964).

[20] N. F. Johnson and M. C. Payne, Exactly Solvable Model of Interacting Particles in a Quantum Dot, Phys. Rev. Lett. 67, 1157 (1991).

[21] H. R. Post, Many-particle systems: Derivation of a shell model, Proc. Soc. A 66, 649 (1953).

[22] N. T. Zinner and A. S. Jensen, Nuclear $\alpha$-particle condensates: Definitions, occurrence conditions, and consequences, Phys. Rev. C 78, 041306 (2008).

[23] Z.-L. Wang, A. M. Wang, Y. Yang, and X. C. Li, Exact eigenfunctions of $N$-body system with quadratic pair potential, Commun. Theor. Phys. 58, 639 (2012).

[24] C. Schilling, D. Gross, and M. Christandl, Pinning of Fermionic Occupation Numbers, Phys. Rev. Lett. 110, 040404 (2013).

[25] C. Schilling, Natural orbitals and occupation numbers for harmonium: Fermions versus bosons, Phys. Rev. A 88, 042105 (2013).

[26] A. S. Jensen, T. Kjærgaard, M. Thøgersen, and D. V. Fedorov, Eigenvalues of the one-body density matrix for correlated condensates, Nucl. Phys. A 790, 723c (2007).

[27] J. R. Armstrong, N. T. Zinner, D. V. Fedorov, and A. S. Jensen, Analytic harmonic approach to the $N$-body problem, J. Phys. B 44, 055303 (2011).

[28] M. Gajda, Criterion for Bose-Einstein condensation in a harmonic trap in the case with attractive interactions, Phys. Rev. A 73, 023603 (2006). 


\section{CHRISTIAN SCHILLING AND ROLF SCHILLING}

[29] R. B. Laughlin, Anomalous Quantum Hall Effect: An Incompressible Quantum Fluid with Fractionally Charged Excitations, Phys. Rev. Lett. 50, 1395 (1983).

[30] See Supplemental Material at http://link.aps.org/supplemental/ 10.1103/PhysRevA.93.021601 for technical details on the 1$\mathrm{RDM}$, its eigenvalue equation in the strong-coupling regime, and the effective potential.

[31] J. Pipek and I. Nagy, Measures of spatial entanglement in a two-electron model atom, Phys. Rev. A 79, 052501 (2009).

[32] C. Schilling and R. Schilling, Duality of reduced density matrices and their eigenvalues, J. Phys. A 47, 415305 (2014).

[33] P. Kościk, The von Neumann entanglement entropy for Wignercrystal states in one dimensional $N$-particle systems, Phys. Lett. A 379, 293 (2015).

[34] F. Calogero, Ground state of a one-dimensional $N$-body system, J. Math. Phys. 10, 2197 (1969).

[35] F. Calogero, Solution of the one-dimensional $N$-body problems with quadratic and/or inversely quadratic pair potentials, J. Math. Phys. 12, 419 (1971).

[36] B. Sutherland, Quantum many-body problem in one dimension: Ground state, J. Math. Phys. 12, 246 (1971).
PHYSICAL REVIEW A 93, 021601(R) (2016)

[37] B. Sutherland, Exact results for a quantum many-body problem in one dimension, Phys. Rev. A 4, 2019 (1971).

[38] B. Sutherland, Exact results for a quantum many-body problem in one dimension. II, Phys. Rev. A 5, 1372 (1972).

[39] M. Weidemüller and C. Zimmermann, Interactions in Ultracold Gases: From Atoms to Molecules (Wiley, New York, 2011).

[40] I. Bloch, J. Dalibard, and W. Zwerger, Many-body physics with ultracold gases, Rev. Mod. Phys. 80, 885 (2008).

[41] C. Chin, R. Grimm, P. Julienne, and E. Tiesinga, Feshbach resonances in ultracold gases, Rev. Mod. Phys. 82, 1225 (2010).

[42] A. G. Truscott, K. E. Strecker, W. I. McAlexander, G. B. Partridge, and R. G. Hulet, Observation of Fermi pressure in a gas of trapped atoms, Science 291, 2570 (2001).

[43] G. Zürn, F. Serwane, T. Lompe, A. N. Wenz, M. G. Ries, J. E. Bohn, and S. Jochim, Fermionization of Two Distinguishable Fermions, Phys. Rev. Lett. 108, 075303 (2012).

[44] E. H. Lieb, Density functionals for Coulomb systems, Int. J. Quantum Chem. 24, 243 (1983).

[45] R. G. Parr and W. Yang, Density-Functional Theory of Atoms and Molecules (Oxford University Press, New York, 1994). 